\title{
Postoperative Cognitive Dysfunction: An Updated Review
}

Khalil S, Roussel J, Schubert A $^{*}$ and Emory L

Ochsner Health System, Anesthesiology, USA

*Corresponding author: Armin Schubert, Ochsner Health System, Anesthesiology, 1514 Jefferson Hwy Hospital, 2nd FI, New Orleans, Louisiana 70121, USA, Tel: 5048424221; Fax: 5048426086; E-mail: aschubert@ochsner.org

Received date: March 04, 2015; Accepted date: April 30, 2015; Published date: May 03, 2015

Copyright: $\odot 2015$ Khalil S et al. This is an open-access article distributed under the terms of the Creative Commons Attribution License, which permits unrestricted use, distribution, and reproduction in any medium, provided the original author and source are credited.

\begin{abstract}
Post operative Cognitive Dysfunction is a condition that follows immediately after a major surgery or any other serious health ailment. It shows significant clinical and social impact on the patient that may often result in either death or impairment, disabling the individual to enter into the job market. While $30 \%$ to $40 \%$ of postoperative patients, particularly elders are affected immediately after getting discharged from the hospital, $5 \%$ to $12 \%$ of patients are affected after three months.
\end{abstract}

The origin and the reasons for POCD are not clearly known and there are no universally acceptable evidence based management strategies to cope with this emergency. There is no consensus among experts across the globe on how to treat these kinds of disorders and the definition for the Post Operative Cognitive Dysfunction is missing in the existing literature, leading to further confusion.

This article attains importance as tries to analyze the most recent theories of pathogenesis and the risks that may follow Post operative Cognitive Dysfunction by reviewing articles published on this subjects for the past 60 years, starting from 1955 to 2014 in PubMed and Google Scholar and suggests relevant measures on how to cope with it.

Keywords: Cognitive dysfunction; Anesthesia; Cognition; Surgery

\section{Introduction}

Postoperative cognitive dysfunction (POCD) is an important condition that may follow surgery [1] or other procedures [2] and has significant clinical and social impacts. It is associated with increased risk of mortality, premature departure from the labor market and social dependency [3]. It affects a significant percentage of postoperative patients, of all age groups [4,5], particularly the elderly [6], with an estimated incidence of $30-40 \%$ at hospital discharge and $5-12 \%$ at 3 months for noncardiac surgeries [7]. The pathogenesis is not fully understood and effective, evidence-based management strategies are not currently described. There are no universally accepted guidelines for the diagnosis and treatment of POCD, and there have been few consensus statements on the subject. For example, there was a consensus statement published in Annals of Thoracic Surgery in 1995 which highlighted the recommended battery of neuropsychological testing to assess for POCD [8], although this has not been adopted consistently in the subsequent literature. The definition of POCD is neither included in the Diagnostic and Statistical Manual (DSM-V) nor in International Classification of Diseases [ICD-10], and the clinical awareness of anesthesiologists is still incomplete. In this review, we shed light on the most recent theories of pathogenesis and risk factors, experimental and clinical treatments, and recommendations for clinicians based on the available data.

\section{Methods}

A comprehensive search was done using PubMed and Google Scholar. Articles were extracted over a period of 60 years from 1955 through November 2014. However, most are dated from 1998 onward. We also selected and examined appropriate references from the gathered articles. Key words included, but were not limited to the following: POCD review article, POCD in the elderly, middle aged, pediatric population, assessment of cognitive impairment/POCD, pathophysiology of POCD, management of POCD, anesthetics and POCD, anesthetics and Alzheimer's disease, general versus regional anesthesia and POCD , POCD and depth of anesthesia, pharmacology and POCD; Isoflurane, sevoflurane, desflurane anesthesia and POCD; Ketamine, propofol, benzodiazepines and POCD, biomarkers and POCD, neuroinflammation and POCD, Total Intravenous Anesthesia [TIVA] and POCD, dexmedetomidine and POCD.

\section{Awareness and assessment of the problem}

As early as 1955 and 1957 postoperative neurocognitive changes were reported to occur after noncardiac and cardiac surgeries, respectively $[9,10]$. Investigational activity regarding this topic culminated with publication of large multicenter prospective randomized trial results by the International Study of PostOperative Cognitive Dysfunction group (ISPOCD), some of which included long term follow-ups [11]. Since then, interest in this topic has continued in the clinical scientific perioperative community as evidenced by a steady stream of insightful papers.

Currently there is no standardized definition of POCD; the closest term in ICD-10 is "mild cognitive impairment". POCD is simply a deviation from normal cognition; however the definition of the extent of deviation varies among studies. Furthermore, the cognitive changes in POCD are subtle and must be confirmed by a battery of neuropsychological tests. Hence the diagnosis cannot be made on purely clinical grounds. The same domains that are assessed in dementia are also assessed in POCD. They include learning and 
memory, language, executive function, complex attention, perceptualmotor function and social cognition [12].

The accurate assessment of POCD is difficult with a variety of contributing elements [13]. The most often mentioned are variability of examiners, different definitions of POCD, interval between test sessions, mood changes and anxiety, pain, sleep-deprivation, pharmacologic effects, failure of some patients to complete the studies, language and cultural problems, timing of postoperative assessment, differences in the tests used, surrounding environments, dissimilar exclusion criteria, and statistical design. Most well designed studies include a baseline assessment one week before; they follow up at one week and at three months after surgery; occasionally follow-ups as long as several years after surgery are reported. Large sample size, inclusion of non-surgical control groups, and using full set of neuropsychological tests are acknowledged as adding validity to study design. The Mini-Mental Status Examination (MMSE) is useful as a surrogate for clinical but not investigational purposes. Though it has sensitivity of $87 \%$ and specificity of $82 \%$ and is less time consuming than neuropsychological testing [14], it lacks sensitivity for detecting mild dementia [15].

Several investigator groups have searched for biomarkers of POCD for diagnostic or prognostic purposes. For example, Apolipoprotein E4 is a known marker for neurodegenerative diseases, but was not found useful for assessment of POCD [16]. In a pilot study, Price et al. evaluated whether preoperative MRI neuroimaging might predict POCD in nondemented patients. They found that reduced hippocampal/entorhinal volume predicted memory change; moreover, the degree of leukoaraiosis and lacunar volume predicted decrements in executive function [17]. Similarly, increased levels of IL-6 and S-100B correlated well with the incidence of POCD [18]. Other markers that have been studied include tau protein, malonaldehyde, melatonin level, neutrophil-lymphocyte ratio, and aspartic acid level, some with promising results [19-24]. However, further study is needed to prospectively validate how useful these biomarkers might be to identify high risk patients or those who might benefit from prophylactic or therapeutic intervention.

\section{Pathophysiology, etiology and risk factors}

Much of the available work suggests that causative factors of POCD could include the surgical experience [including the effect of hospitalization - recently subsumed under the term "post-hospital syndrome [25] or potentially neurotoxic effects of anesthesia [26]. Therefore, in some studies, POCD is explained neither by surgery nor by anesthesia alone. Indeed, postoperative cognitive improvements relative to preoperative function has even been reported after surgery and anesthesia $[27,28]$. This suggests that other factors are involved in the pathogenesis of POCD such as the patient's own pathological state and subsequent systemic inflammatory conditions. For example, a Japanese prospective study [29], showed that preoperative existence of low grey matter volume and white matter lesions on MRI were associated with higher incidence of POCD after elective cardiac surgery. In a study using standardized assessment tools and good statistical design, Evered et al. compared the occurrence of POCD after CABG [on-pump; general anesthesia], coronary angiography [sedation only], and total hip arthroplasty [spinal and light general anesthesia]; the incidence of POCD after 3 months was $16 \%, 21 \%$ and $16 \%$ respectively. Surprisingly, the least invasive procedure performed only with sedation was associated with the highest incidence of POCD. This suggests that neither the intensity of surgical or procedural intervention nor the type of anesthesia alone can predict the occurrence of POCD [30].

It has been proposed that the surgical experience might induce POCD through surgery-induced systemic inflammation via activation of the immune system and release of proinflammatory cytokines [e.g. IL-1B, IL-6, TNF]. The latter are thought to violate the integrity of blood brain barrier and induce inflammation in the hippocampus, an area known to mediate memory and learning [31,32]. This response is even more exaggerated in the diseased brain [33-35]. General anesthetics could potentially lead to memory impairment through hyperphosphorylation of tau-protein, an important protein involved in the pathogenesis of Alzheimer's disease. Tau hyperphosphorylation has been attributed to anesthesia-induced hypothermia [36,37]. However, a recent study showed that sevoflurane induced memory deficits even in normothermic mice [38]. Moreover, earlier studies attributed neuroinflammation to surgery only $[39,40]$, but a more recent study has suggested anesthetic mediation as well. For example repeated exposures to anesthetics have more influence on learning, memory, and function than a single exposure [41]. Therefore, POCD or at least the components of POCD relating to memory function may also be generated through a direct effect of anesthetics on brain cells and circuits.

Furthermore, anesthetics may have a direct toxic effect on neural structures. For example, high concentrations of inhalational anesthetics can increase blood brain barrier permeability [42]. In a recent study [43], isoflurane disrupted hippocampal Neuregulin 1ErbB4 in mice, which plays a key role in synaptic plasticity, and impairs learning and hippocampal long-term potentiation [LTP] [44]. Other studies in neonatal monkeys showed that prolonged ketamine infusion causes neurodegeneration and neuroapoptosis [45,46]; the same was found with isoflurane [47]. Dong et al. observed that sevoflurane induced apoptosis and elevated levels of B-site amyloid precursor protein-cleaving enzyme [48]. Taken together, these and many other findings lead to a consensus statement on the possible link between Alzheimer's disease and anesthesia which states " there is sufficient evidence at multiple levels to warrant further and more definitive investigation of the onset and progression of Alzheimer's disease and neurodegeneration after anesthesia and surgery" [49]. Currently, however, there remains no concrete evidence to support a direct causative relationship of anesthesia with regard to initiation or progression of Alzheimer's disease. Interestingly, The ISPOCD group followed up patients from ISPOCD-1 and ISPOCD-2 for a median duration of 11 years without finding a significant relationship between anesthesia, POCD and dementia [50].

The prospect that general anesthesia [GA] may be neurotoxic led researchers to compare patients receiving GA with those having regional anesthesia [RA] or neuroaxial anesthesia. A review of seventeen clinical studies $[51,52]$ failed to find a significant difference in the incidence of POCD after GA compared to RA. The main criticism of these studies is that RA is often combined with sedation, frequently deep sedation. Initially regional anesthesia without deep sedation [i.e. BIS $>80$ was shown to result in a substantially lower occurrence of delirium compared with [53]. Because delirium and POCD are linked, it was reasonable to assume a similar relationship for POCD. Therefore, Silbert et al. [2] prospectively compared the incidence of POCD after GA for Extracorporeal Shockwave Lithotripsy [ESWL] with the use of spinal anesthesia without sedation. The incidence of POCD was almost three times as high after spinal anesthesia. At 1 week, POCD was $11.9 \%$ after spinal vs. $4.1 \%$ after GA; 
at 3 months the incidence was $19.6 \%$ and $6.8 \%$ for spinal and GA respectively. The investigators could not conclude superiority of one technique over the other; clearly POCD could not be avoided through the use of RA without sedation. This and other findings after minimally invasive interventions point to a likely effect of hospitalization on patient functional status. Physician deconditioning has long been recognized as a consequence of hospital stays [54,55].

Depth of GA has been investigated as a contributing factor for POCD. Two studies suggested that deeper level of anesthesia monitored by BiSpectral Index [BIS] to values between 30-40 result in better cognitive outcome than lighter values [i.e. 50-60] [56,57]. The main criticisms of this work are relatively small sample sizes and that assessment for POCD was done in the early postoperative period [4-6 weeks and 5 days, respectively]. In addition, only 3 neuropsychological tests were used for assessment in the first study instead of a full battery of tests. The CODA trial [COgnitive Dysfunction after Anesthesia] is a prospective randomized study of 921 patients. The patients were divided into a BIS-titrated group with a target of 40-60 versus BISblinded group. The BIS-titrated group had less delirium, less POCD and decreased anesthetic delivery than the BIS-blinded group, where BIS values were lower than in the BIS-guided group [58]. Another large prospective study found an association between low BIS values and higher incidence of delirium but not POCD [59]. More alarmingly, multiple studies have linked low BIS values with mortality $[60,61]$. A large retrospective study from Cleveland Clinic reported an association between a composite "triple low state" [low BIS, low blood pressure, and low Minimal Alveolar Concentration] and mortality [62]. On the other hand, investigators from Duke University could not confirm this association of mortality with the "triple low state" in a large retrospective study [63]. Although the "triple low state" studies include depth of anesthesia as one of the three components, it is unclear to what extent any one parameter contributed to the observed associations. Results from a pilot study from New Zealand and Australia, conducted in preparation for a larger clinical trial, indicate that "expected higher complication and mortality will be present with low BIS values" [64]. While the evidence for an association between depth of anesthesia and incidence of POCD is far from conclusive, currently available evidence seems to suggest that deeper levels of anesthesia may be associated with less than optimal outcome. Clinicians may well consider adjusting anesthetic dose to a level minimally capable of achieving the goals of anesthetic management, prioritized on surgical and patient factors. Interestingly, a recent survey of Australian anesthesiologists indicates that most anesthesiologists who utilize depth of anesthesia [DoA] electroencephalogram [EEG] monitors do so in an attempt to prevent awareness; the survey also suggests that practitioners may in the future look toward DoA monitoring as a means to improve recovery and minimize complications. Survey results showed that $62 \%$ of respondents thought that DoA monitors allowed them to use less anesthetic. This capability provided benefits that were thought to include "faster emergence, less morbidity, and improved hemodynamic stability" [65] .

The type of GA has been examined as a contributing factor to POCD. Cai and co-workers conducted a large single center casecontrolled study to investigate the association between Apolipoprotein $\mathrm{E} 4$ and POCD in an elderly population. They compared surgical patients receiving total intravenous anesthesia [TIVA] with others receiving general inhalational anesthesia [GIA]. They found that patients who received GIA had a significant decrease in postoperative MMSE scores on postoperative day 3 when compared to preoperative baseline; their postoperative MMSE scores were also lower than those of the TIVA group. In the GIA group, decreases in MMSE score were related to the presence of the Apo E4 allele. This association was not found in the TIVA group [66]; this finding suggests an interaction between type of anesthetic exposure and genetic predisposition in the development of POCD. In another study, type of anesthetic exposure, neuro-inflammation, and POCD were examined in patients specifically undergoing on-pump CABG. The TIVA group received propofol and fentanyl and the GIA group received sevoflurane and fentanyl. Neuro-inflammation was assessed with the serum biomarker protein S100B. The TIVA group had higher levels of S100B as well as lower MMSE and Montreal Cognitive Assessment [MoCa] scores when compared to the GIA group [67]. The differences in the results from these two studies are difficult to explain. Potential confounders include type of surgery, the patient population, or the individual risk factors of the patients [e.g. ApoE4 presence, etc.]. Furthermore, the investigator teams used different tools to detect POCD [MMSE alone and MMSE plus MoCA], and both evaluated their patients only early in the postoperative period. These studies highlight the difficulties that may arise from when standards for testing and timing of POCD evaluation are not adopted, as outlined in the consensus statement mentioned previously [8].

Old Age is considered a major risk factor for POCD and is universally commented on in the available literature. Strom et al summarized seven mechanisms to explain the higher incidence of POCD in older subjects [68]. This includes decreased brain volume, decreased density of the blood-brain barrier, decreased neurogenesis, decreased baseline cognition, decreased cognitive reserve, increased likelihood of inflammation, and cerebrovascular disease. Others risk factors for POCD include low level of education, preexisting cerebrovascular disease, noisy hospital environment, metabolic syndrome and systemic inflammatory states, duration of anesthesia, respiratory and infectious complications, male gender, genetic polymorphism, pain, longer hospital stay, more invasive complicated surgery, diabetes and hyperglycemia [11,52,58,69-74]. Hypoxia and hypotension are mechanisms that could induce cerebral ischemia. They were examined in ISPOCD1 study [11] and were not found to be significant risk factors for POCD. However, direct measurement of cerebral oxygen desaturation predicts POCD in cardiac [75] and noncardiac surgery and might be useful in selected cases [76,77]. For investigative work it is important to account for such risk factors in the development of one's study design. Until effective preventative measures and therapeutic interventions have been identified, it is difficult to envision their use by practitioners in clinical decision making.

Showers of microemboli during cardiac surgery, especially during cardiopulmonary bypass, were thought to represent a mechanism of subtle ischemia and POCD [78]. MRI is a useful modality for detecting cerebral ischemia and microinfarcts caused by micro-emboli. Knipp et al. [79] prospectively examined 39 patients undergoing CABG by neuropsychological testing and MRI preoperatively and up to 3 years after the surgery. They found ischemic cerebral lesions in $51 \%$ of patients, but they failed to find an association between these lesions and POCD. Given the small population studied, further large scale studies are needed to confirm these findings and their clinical significance. To wit, studies using Transcranial Doppler [TCD] did not find a relationship between embolic load and occurrence of POCD $[80,81]$. Indeed, an association between the number of emboli and POCD was absent in both cardiac and orthopedic surgery $[82,83]$ as well as coronary angiography procedures [84]. A meta-analysis by 
Page 4 of 8

Marasco et al. [85] failed to find a higher incidence of POCD after offpump cardiac surgery [less emboli] versus on-pump cardiac surgery [more emboli], suggesting that microemboli do not represent a significant pathogenic factor.

Interestingly, a recent meta-analysis concluded that cardiac surgery results in postoperative cognitive improvement relative to preoperative baseline testing. This suggests that improvement of myocardial ischemia may improve cognitive dysfunction [86]. Myocardial ischemia has emerged as an important potential risk factor for POCD. Zhu et al. [87] demonstrated that transient myocardial ischemia [induced by ligation of the left anterior descending artery for 30 minutes] caused in vivo cognitive dysfunction evidenced by impaired long term potentiation [LTP] and increased expression of inflammatory biomarkers. This effect has been attenuated by preconditioning with sevoflurane. LTP impairment did not occur after a sham procedure. Myocardial ischemia-reperfusion injury therefore likely plays a significant role in the occurrence of POCD after cardiac surgery.

\section{Therapeutic Interventions: Experimental and clinical trials}

Anti-inflammatory and neuroprotective therapies have been investigated for their potential to prevent POCD. In one animal study, neutralizing antibody to alarmin, a high-mobility group box 1 protein involved in neuro-inflammation, prevented the inflammatory response and decreased the incidence of memory deficits [88]. Similarly, resolvins are potent endogenous lipid mediators that are biosynthesized during the resolution phase of inflammation. Aspirin has been shown to trigger resolvin D1 production which in turn attenuated the effect of memory decline [89]. Minocycline mitigated isoflurane-induced cognitive impairment in aged rats [90]. Atorvastatin, an anti-inflammatory drug, attenuated in vivo reduction of the hippocampal-dependent fear response induced by surgery [91]. An animal study involving 344 rats suggests that amantadine attenuates learning and memory impairment after surgical intervention [92]. Dexmedetomidine attenuated isoflurane-induced neurocognitive impairment in developing neonatal rats [93].

In a recent clinical trial, magnesium failed to improve cognitive function after cardiac surgery [94]. Low dose bolus ketamine may attenuate POCD after cardiac surgery [95]. Two studies, one experimental and one clinical, demonstrated the role of intranasal insulin in maintenance of normoglycemia which lead to decreased tau hyperphosphorylation and improved cognitive outcome [96,97]. Because of the association of cognitive dysfunction with sleep deprivation and resultant melatonin fluctuations, breast cancer patients were pretreated with melatonin; this resulted in improved sleep efficiency but not cognitive function after breast cancer surgery [98]. The effects of dexamethasone on the incidence of POCD were reported with varying results. One study [99] reported a beneficial effect of low dose $[0.1 \mathrm{mg} / \mathrm{kg}]$ dexamethasone on the incidence of POCD but a harmful effect with high dose $[0.2 \mathrm{mg} / \mathrm{kg}]$ administration. Another study [100] reported that high dose $[1 \mathrm{mg} / \mathrm{kg}]$ dexamethasone is not beneficial. Yet another study showed that $8 \mathrm{mg}$ of IV dexamethasone can significantly decrease the incidence of POCD in elderly patients who undergo cardiac surgery. The effect was greater when combined with superficial anesthesia [101]. Other drugs including ondansetron, ketoprofen, ACE inhibitors, and resveratrol could have beneficial effects [102-105].

\section{Assessment of current anesthetics}

As a general rule, there is no clear superiority of any one the currently available anesthetics with respect to development of POCD. Bilotta et al reviewed 16 drugs [106], the incidence of POCD didn't differ between patients and controls in thiopental, ketamine, propofol and xenon, with conflicting results for lidocaine, ketamine and magnesium sulfate. Some studies suggest there may be a beneficial effect of desflurane over sevoflurane over isoflurane [107,108]. In a clinical study comparing spinal anesthesia plus desflurane, versus spinal anesthesia plus isoflurane versus spinal anesthesia alone, POCD incidence at one week was lowest in the desflurane group [109]. Repeated exposure of neonatal mice [ 3 times versus one time] didn't induce neuroinflammation or cognitive dysfunction with desflurane or sevoflurane, respectively [110]. Benzodiazepines are known to affect concentration in elderly patients [111], however a clear relation between their serum concentration and POCD has not been proven [112].

Delirium is another condition that is often looked at in comparison to POCD. Dexmedetomidine, compared to midazolam, decreased delirium and facilitated early extubation in ICU mechanically ventilated patients [113]. Despite this finding, delirium and POCD are separate entities. A relationship has yet to be fully elucidated and properly studied, and it is still unclear if strategies that reduce delirium can also reduce POCD [114]. One study reviewed examined delirium in mechanically ventilated ICU patients as a predictor for long term cognitive impairment. The majority of patients' evaluated were critically ill with diagnoses of sepsis and ARDS. The patients were evaluated with 9 neuropsychiatric tests administered by a neuropsychiatrist at 3 months and 12 months. They found that delirium was in fact an independent predictor of long term cognitive impairment and also noted that an increase in the duration of delirium also increased long term cognitive impairment [115]. This study suggests a relationship between delirium and cognitive impairment, but this is also another example of investigators using different definitions/evaluation tools for cognitive impairment. In another study that evaluated patients after cardiac surgery, the relationship between delirium and long term cognitive and functional outcomes was examined. In this study, patients diagnosed with delirium based off Diagnostic and Statistical Manual for Mental Disorders [DSM-IV] were given a questionnaire 1-1.5 years after having cardiac surgery. Sleep disturbance was the only category evaluated noted to have a statistically significant difference between patient's with post operative delirium and without delirium. Mortality, readmission rates, memory/ concentration, and dependency for activities of daily living [ADLs] all had a negative tendency in the delirium groups, but there was no statistically significant difference. Of note, this study was limited by a small sample size and the use of a mailed questionnaire [116]. The results of this study suggest a need for further study in this area to truly determine if a relationship between delirium and cognitive impairment exists. A Taiwanese study evaluated similar data in orthopedic surgery patients. They utilized a Comprehensive Geriatric Assessment pre-operatively and 24 hours post-operatively. This assessment looked at visual/hearing deficits, polypharmacy, depression, nutrition, comorbidity, pain, cognitive function, and ADLs. After discharge at 1, 3, 6, and 12 months, the patients received a phone call to assess their ability to perform ADLs. This study showed worsening of scores regarding independence with ADLs at 6 and 12 months in patients with delirium, as well as even worse scores in patients with delirium and cognitive impairment [117]. This study calls for early identification of cognitive impairment and the 
implementation of strategies to prevent delirium. Another study looked at neuroimaging in relation to both delirium and cognitive impairment. Patients that were diagnosed with delirium in the hospital were evaluated at 3 and 12 months post discharge. The investigators evaluated 5 indices: immediate memory, visuospatial, language, attention, and delayed memory. The patients also underwent an MRI scan at discharge and 3 months later. Specific areas of the brain were examined for fractional anisotropy which is a calculation done with diffusion tensor imaging to evaluate axonal integrity. The study showed that longer duration of delirium was associated with white matter disruption on imaging, and white matter disruption was associated with worsened cognitive outcomes at 12 months [118]. These studies all suggest that further study into the relationship between POCD and delirium needs to be investigated further. Unfortunately, these studies also highlight the need for standardization of evaluation tools for cognitive impairment if more progress into understanding POCD is hoped to be achieved.

\section{Recommendations}

Despite significant and copious research on the subject, Postoperative Cognitive Dysfunction remains poorly defined and poorly understood. While many potential explanations are suggested, a definitive pathophysiology has not been described, and a direct causal relationship has not been firmly established between the disease and any suggested insult. Furthermore, no definitive peri-operative or, more specifically, anesthetic strategy has been shown to definitively improve the incidence or severity of POCD.

Despite this, there are several intriguing possibilities both for future research and for application of studied practices to attenuate the problem. We strongly advocate pre-operative discussion with patients and their families regarding the possibility of post-operative cognitive dysfunction in at-risk groups.

Pre-operative discussion with the patient's family is highly recommended to discuss the potential for POCD to occur. It is important to stress that there are various causes of this phenomenon, including the surgery itself, and also that the effect is temporary. This is especially important in elderly patients and/or patients with baseline dementia or cognitive deficits. A MMSE can be considered before and after surgery for patients that are at risk for POCD [elderly, patients with baseline dementia or cognitive deficits, etc] in order to establish a baseline and monitor for the occurrence of POCD, respectively. The routine use of midazolam or other benzodiazepines except in populations that are felt to be at risk should be avoided unless strongly indicated. Meperidine should also be avoided. While it is unclear whether it definitely improves outcome, minimally invasive surgery or laparoscopic surgery should be advocated to decrease the extent of inflammatory response and subsequently reduce the chances of developing POCD.

Hypothermia in conjunction with inhalational anesthetics enhances tau phosphorylation in animal studies which leads to increased memory deficits. Hyperthermia is also recognized as harmful in cardiac and brain surgeries. Hence, maintaining normothermia is strongly advocated. Hyperglycemia appears to be associated with POCD, and worsens outcome in neurosurgery and cardiac surgery. Normoglycemia should be maintained.

There is conflicting evidence regarding depth of anesthesia and post-operative congnitive dysfunction. Some evidence suggests the potential for higher rates of POCD with deeper anesthesia, while more recent evidence seems to suggest that anesthesia titrated to BIS-scores between 40-60 improves the likelihood of POCD. It is recommended to avoid the use of anesthetics for non-anesthetic purposes, e.g. treating hypertension by increasing the dose of inhaled anesthetic. It is important to pay attention to anesthetic requirements on a patient by patient basis. DoA monitors may be used in the future to help providers use less anesthetics per case and therefore improve outcomes. Some studies suggest that for inhalational anesthetics, desflurane is superior to sevoflurane, and sevoflurane is superior to isoflurane in reducing POCD. Dexmedetomidine may be the best current sedating agent for the mechanically ventilated patient that could enhance earlier release and decrease the incidence of delirium, but it is still unclear if these strategies also decrease the incidence of POCD. Ketamine was shown to reduce POCD in cardiac patients in one study and further research is likely indicated here. Epidural analgesia may be better than parenteral analgesia during the inhospital recovery period [119], but, surprisingly, spinal anesthesia was not better but actually worse than general anesthesia for reducing post-operative cognitive dysfunction.

Obviously, myocardial ischemia should be avoided in general for several reasons, but recently gained insight indicates that maintenance of normal cardiac perfusion may reduce the chance for POCD. Furthermore, monitoring of cerebral oxygenation may be useful in select patient populations, although this has not been shown to reduce the incidence of the disease.

While both anesthesia and surgery have been associated with POCD, there are other factors that appear to contribute as well. For example, prolonged hospital stays, sleep deprivation in the hospital, and postoperative pain may all contribute to POCD. Minimizing length-of-stay, carefully managing post-operative pain, and improving patient sleep-efforts may help with this disease. Implementation of fast-track policy in orthopedic surgery could decrease early POCD [120].

\section{References}

1. Coburn M, Fahlenkamp A, Zoremba N, Schaelte G (2010) Postoperative cognitive dysfunction: Incidence and prophylaxis. Anaesthesist 59: 177-184.

2. Silbert BS, Evered LA, and Scott DA. Incidence of postoperative cognitive dysfunction after general or spinal anaesthesia for extracorporeal shockwave lithotripsy. British Journal of Anaesthesia Page 1 of 8.

3. Steinmetz J, Christensen KB, Lund T, Lohse N, Rasmussen LS; ISPOCD Group (2009) Long-term consequences of postoperative cognitive dysfunction. Anesthesiology 110: 548-555.

4. Tim Johnson, Terri Monk, Lars S. Rasmussen, Hanne Abildstrom, Peter Houx, et al. (2002) Postoperative Cognitive Dysfunction in Middle-aged Patients. Anesthesiology 96.

5. WarnerVoigt DO, Olson MD, Sprung J, Weaver Al, Schroeder DR, et al. (2011) Cognitive and Behavioral Outcomes After Early Exposure to Anesthesia and Surgery.Pediatrics 128.

6. Canet J, Raeder J, Rasmussen LS, Enlund M, Kuipers HM, et al. (2003) Cognitive dysfunction after minor surgery in the elderly. Acta Anaesthesiol Scand 47: 1204-1210.

7. Monk TG, Weldon BC, Garvan CW, Dede DE, van der Aa MT, et al. (2008) Predictors of cognitive dysfunction after major noncardiac surgery. Anesthesiology 108: 18-30.

8. Murkin JM, Newman SP, Stump DA, Blumenthal JA (1995) Statement of consensus on assessment of neurobehavioral outcomes after cardiac surgery. Ann Thorac Surg 59: 1289-1295.

9. Bedford PD (1955) Adverse cerebral effects of anaesthesia on old people. Lancet 269: 259-263. 
10. Boshes B, Priest Ws, Yacorzynski Gk, Zaks Ms (1957) The neurologic psychiatric and psychologic aspects of cardiac surgery. Med Clin North Am 41: 155-169.

11. Moller JT1, Cluitmans P, Rasmussen LS, Houx P, Rasmussen H, et al. (1998) Long-term postoperative cognitive dysfunction in the elderly ISPOCD1 study. ISPOCD investigators. International Study of PostOperative Cognitive Dysfunction. Lancet 351: 857-861.

12. Shalden MF, Dekoksky ST, Schmader KE (2014) April F Eichler. Evaluation of cognitive impairment and dementia.

13. Rasmussen LS, Larsen K, Houx P, Skovgaard LT, Hanning CD, et al. (2001) The assessment of postoperative cognitive function. Acta Anaesthesiol Scand 45: 275-289.

14. Crum RM, Anthony JC, Bassett SS, Folstein MF (1993) Population-based norms for the Mini-Mental State Examination by age and educational level. JAMA 269: 2386-2391.

15. Freidl W, Schmidt R, Stronegger WJ, et al. (1996) Mini mental state examination: influence of sociodemographic, environmental and behavioral factors and vascular risk factors. J Clin Epidemiol 49:73.

16. McDonagh DL, Mathew JP, White WD, Phillips-Bute B, Laskowitz DT, et al. (2010) Newman, for the Neurologic Outcome Research Group. Cognitive Function after Major Noncardiac Surgery, Apolipoprotein E4 Genotype, and Biomarkers of Brain Injury. Anesthesiology 112: 852-859.

17. Price CC, Tanner JJ, Schmalfuss I, Garvan CW, Gearen P, et al. (2014) A pilot study evaluating presurgery neuroanatomical biomarkers for postoperative cognitive decline after total knee arthroplasty in older adults. Anesthesiology 120: 601-613.

18. Peng L, Xu L, Ouyang W (2013) Role of Peripheral Inflammatory Markers in Postoperative Cognitive Dysfunction (POCD): A MetaAnalysis.

19. Xie Z, McAuliffe S, Swain CA, Ward SAP, Crosby CA, et al. (2013) Cerebrospinal Fluid $A ß$ to Tau Ratio and Postoperative Cognitive Change. Annals of Surgery 258.

20. Mu-Huo J, Hong-Mei Y, Guang-Fen Z, Xiao-Min L, Lin D, et al. (2013) Changes in plasma and cerebrospinal fluid biomarkers in aged patients with early postoperative cognitive dysfunction following total hipreplacement surgery. J Anesth 27: 236-242.

21. Halazun HJ, Mergeche JL, Mallon KA, Connolly ES, Heyer EJ (2014) Neutrophil-lymphocyte ratio as a predictor of cognitive dysfunction in carotid endarterectomy patients. J Vasc Surg 59: 768-773.

22. Rong Hu, Dong Huang, Jianbin Tong, Qin Liao, Zhonghua Hu, et al. (2014) Aspartic acid in the hippocampus: a biomarker for postoperative cognitive dysfunction. NEURAL REGENERATION RESEARCH.

23. Cheng Q, Wang J, Wu A, Zhang R, Li L, et al. (2013) Can urinary excretion rate of 8-isoprostrane and malonaldehyde predict postoperative cognitive dysfunction in aging?. Neurol Sci 34: 1665-1669.

24. Wu Y, Wang J, Wu AS, Yue Y (2014) Do fluctuations in endogenous melatonin levels predict the occurrence of postoperative cognitive dysfunction (POCD)?. International Journal of Neuroscience.

25. Krumholz HM (2013) Post-hospital syndrome--an acquired, transient condition of generalized risk. N Engl J Med 368: 100-102.

26. Vlisides P, Xie Z (2012) Neurotoxicity of general anesthetics: an update. Curr Pharm Des 18: 6232-6240.

27. Alosco ML, Spitznagel MB, Strain G, Devlin M, Cohen R, et al. (2014) Improved Memory Function Two Years After Bariatric Surgery. Obesity 22: 32-38.

28. Seminowicz DA, Wideman TH, Naso L, Hatami-Khoroushahi Z, Fallatah S, et al. (2011) Effective Treatment of Chronic Low Back Pain in Humans Reverses Abnormal Brain Anatomy and Function. The Journal of Neuroscience 31: $7540-7550$.

29. Maekawa K, Baba T, Otomo S, Morishita S, Tamura N (2014) Low PreExisting Gray Matter Volume in the Medial Temporal Lobe and White Matter Lesions Are Associated with Postoperative Cognitive Dysfunction after Cardiac Surgery 9: e87375.
30. Evered L, Scott DA, Silbert B, and Maruff P (2011) Postoperative Cognitive Dysfunction Is Independent of Type of Surgery and Anesthetic.Anesthesia\& Anelgesia 112: 5 .

31. Riedel B, Browne K, Silbert B (2014) Cerebral protection: inflammation, endothelial dysfunction, and postoperative cognitive dysfunction. Curr Opin Anesthesiol 27: 89-97.

32. Terrando N, Eriksson LI, Ryu JK, Yang T, Monaco C, et al. (2011) Resolving postoperative neuroinflammation and cognitive decline. Ann Neurol 70: 986-995.

33. Cunningham C, Wilcockson DC, Campion S, Lunnon K, Perry VH (2005) Central and systemic endotoxin challenges exacerbate the local inflammatory response and increase neuronal death during chronic neurodegeneration. Journal of Neuroscience 25: 9275-9284.

34. Cunningham C, Campion S, Lunnon K, Murray CL, Woods JF, et al. (2009) Systemic inflammation induces acute behavioral and cognitive changes and accelerates neurodegenerative disease. Biol Psychiatry 65: 304-312.

35. Dilger RN, Johnson RW (2008) Aging, microglial cell priming, and the discordant central inflammatory response to signals from the peripheral immune system. J Leukoc Biol 84: 932-939.

36. Run X, Liang Z, Zhang L, Iqbal K, Grundke-Iqbal I, et al. (2009) Anesthesia induces phosphorylation of tau. J Alzheimers Dis 16: 619-626.

37. Tan W, Cao X, Wang J, Lv H, Wu B, et al. (2010) Tau hyperphosphorylation is associated with memory impairment after exposure to $1.5 \%$ isoflurane without temperature maintenance in rats. Eur J Anaesthesiol 27: 835-841.

38. Le Freche HLN, Brouillette J, Fernandez-Gomez FJ, Patin P, Caillierez R, et al. (2012) An Association to Postoperative Cognitive Impairment. Anesthesiology $116: 42012$.

39. Terrando N, Monaco C, Ma D, Foxwell BM, Feldmann M, et al. (2010) Tumor necrosis factor-alpha triggers a cytokine cascade yielding postoperative cognitive decline. Proc Natl Acad Sci U S A 107: 2051820522.

40. Cibelli M, Fidalgo AR, Terrando N, Ma D, Monaco C, et al. (2010) Role of interleukin-1beta in postoperative cognitive dysfunction. Ann Neurol 68: 360-368.

41. Shen X, Dong Y, Xu Z, Wang H, Miao C, et al. (2013) Selective Anesthesia-induced Neuroinflammation in Developing Mouse Brain and Cognitive Impairment. Anesthesiology $118: 3$.

42. Hua N, Guoc D, Wang H, Xie K, Wang C, et al. (2014) Involvement of the blood-brain barrier opening in cognitive decline in aged rats following orthopedic surgery and high concentration of sevoflurane inhalation. Brain Research 1551: 13-24.

43. Li XM, Su F, Ji MH, Zhang GF, Qiu LL, et al. (2014) Disruption of hippocampal neuregulin 1-ErbB4 signaling contributes to the hippocampus-dependent cognitive impairment induced by isoflurane in aged mice. Anesthesiology 121: 79-88.

44. Uchimoto K, Miyazaki T, Kamiya Y, Mihara T, Koyama Y, et al. (2014) Isoflurane Impairs Learning and Hippocampal Long-term Potentiation via the Saturation of Synaptic Plasticity. Anesthesiology 121: 302-310.

45. Slikker W Jr, Zou X, Hotchkiss CE, Divine RL, Sadovova N, et al. (2007) Ketamine-induced neuronal cell death in the perinatal rhesus monkey. Toxicol Sci 98: 145-158.

46. Zou X, Patterson TA, Divine RL, Sadovova N, Zhang X, et al. (2009) Prolonged exposure to ketamine increases neurodegeneration in the developing monkey brain. Int J Dev Neurosci 27: 727-731.

47. Brambrink AM, Evers AS, Avidan MS, Farber NB, Smith DJ, et al. (2010) Isoflurane-induced Neuroapoptosis in the Neonatal Rhesus Macaque Brain. Anesthesiology 112: 4.

48. Zhang $\mathrm{Y}, \mathrm{Xu} \mathrm{Z}$, Wang H, Dong Y, Shi HN, et al. (2012) Anesthetics Isoflurane and Desflurane Differently Affect Mitochondrial Function, Learning, and Memory. Ann Neurol 71:687-698.

49. Baranov D, Bickler PE, Crosby GJ, Culley DJ, Eckenhoff MF, et al. (2009) Consensus Statement:First International Workshop on Anesthetics and Alzheimer's Disease. Anesth Analg 108: 1627-1630. 
50. Steinmetz J, Siersma V, Kessing LV, Rasmussen LS; ISPOCD Group (2013) Is postoperative cognitive dysfunction a risk factor for dementia? A cohort follow-up study. Br J Anaesth 110 Suppl 1: i92-97.

51. Newman S, Stygall J, Hirani S, Shaefi S, Maze M (2007). Postoperative Cognitive Dysfunction after Noncardiac Surgery: A Systematic Review. Anesthesiology 106: 3 .

52. Rasmussen LS (2006) Postoperative cognitive dysfunction: Incidence and prevention. Best Practice \& Research Clinical Anaesthesiology 20: 315330.

53. Sieber FE, Zakriya KJ, Gottschalk A, Blute MR, Lee HB, et al. (2010) "Sedation depth during spinal anesthesia and the development of postoperative delirium in elderly patients undergoing fracture repair." Mayo Clinic Proceedings 85: 18-26.

54. Hoenig HM, Rubenstein LZ (1991) Hospital-associated deconditioning and dysfunction. J Am Geriatr Soc 39: 220-222.

55. Sager MA, Franke T, Inouye SK, Landefeld CS, Morgan TM, et al. (1996) Functional outcomes of acute medical illness and hospitalization in older persons. Arch Intern Med 156: 645-652.

56. Farag E, Chelune GJ, Schubert A, Mascha EJ (2006) Is Depth of Anesthesia, as Assessed by the Bispectral Index, Related to Postoperative Cognitive Dysfunction and Recovery?. ANESTHESIA \& ANALGESIA 103: 3 .

57. Jianxiong An, Fang Q, Huang CS, Qian X, Fan T, et al. (2011) Deeper Total Intravenous Anesthesia Reduced the Incidence of Early Postoperative Cognitive Dysfunction After Microvascular Decompression for Facial Spasm. J Neurosurg Anesthesiol 23: 12-17.

58. Matthew, Chan, Benny, Cheng, Tatia, et al. (2013) BIS-guided Anesthesia Decreases Postoperative Delirium and Cognitive Decline. J Neurosurg Anesthesiol 25: 1 .

59. Radtke FM, Franck M, Lendner J, Kru“ger S, Wernecke KD et al. (2013) Monitoring depth of anaesthesia in a randomized trial decreases the rate of postoperative delirium but not postoperative cognitive dysfunction. British Journal of Anaesthesia 110: i98-i105.

60. Lindholm ML, Träff S, Granath F, Greenwald SD, Ekbom A, et al. (2009) Mortality within 2 years after surgery in relation to low intraoperative bispectral index values and preexisting malignant disease. Anesth Analg 108: 508-512.

61. Leslie K, Myles PS, Forbes A, Chan MT (2010) The effect of bispectral index monitoring on long-term survival in the B-aware trial. Anesth Analg 110: 816-822.

62. Sessler DI, Sigl JC, Kelley SD, Chamoun NG, Manberg PJ, et al. (2012) Hospital stay and mortality are increased in patients having a "triple low" of low blood pressure, low bispectral index, and low minimum alveolar concentration of volatile anesthesia.Anesthesiology 116: 1195-1203.

63. Kertai MD, White WD, Gan TJ (2014) Cumulative duration of "triple low" state of low blood pressure, low bispectral index, and low minimum alveolar concentration of volatile anesthesia is not associated with increased mortality. Anesthesiology 121: 18-28.

64. Timothy GS, Leslie K, Campbell D, Chan MTV, et al. (2014) A Pilot Study for a Prospective, Randomized, Double-Blind Trial of the Influence of Anesthetic Depth on Long-Term Outcome. anesthes ia \& ana lges ia 118: 5.

65. Ben-Menachem E, Zalcberg D (2014) Depth of anesthesia monitoring: a survey of attitudes and usage patterns among Australian anesthesiologists. Anesth Analg 119: 1180-1185.

66. Cai Y, Haitao H, Pengbin L, Feng G, Dong W, et al. (2012) "Association between the Apolipoprotein E4 and Postoperative Cognitive dysfunction in Elderly Patients Undergoing Intravenous and Inhalational Anesthesia." Anesthesiology 116: 84-93.

67. Likhvantsev V, Grebenchikov O, Shaibakova V, Levikov D, Cherpakov R (2013) "Neuroprotective effect of volatile induction and maintenance of anesthesia (VIMA) vs total intravenous anesthesia (TIVA) during onpump coronary artery bypass grafting (CABG)." European Journal of Anesthesiology 30: 51-54.

68. Strøm C, Rasmussen LS, Sieber FE (2014) Should general anaesthesia be avoided in the elderly? Anaesthesia 69 Suppl 1:35-44.
69. Krenk L, Rasmussen LS, Kehlet H (2010) New insights into the pathophysiology of postoperative cognitive dysfunction. Acta Anaesthesiol Scand 54: 951-956.

70. Hudetz JA, Patterson KM, Iqbal Z (2011) Metabolic syndrome exacerbates short-term postoperative cognitive dysfunction in patients undergoing cardiac surgery: results of a pilot study. J Cardiothorac Vasc Anesth 25: 282-287.

71. Monk TG, Weldon BC, Garvan CW, Dede DE, van der MT, et al. (2008) Gravenstein. Predictors of Cognitive Dysfunction after Major Noncardiac Surgery. Anesthesiology 108: 18-30.

72. Bartels K, McDonagh DL, Newman MF, Mathew JP (2013) Neurocognitive outcomes after cardiac surgery. Curr Opin Anaesthesiol 26: 91-97.

73. Yang C, Zhu B, Ding J, Wang ZG (2014) Isoflurane anesthesia aggravates cognitive impairment in streptozotocin-induced diabetic rats. Int J Clin Exp Med 7: 903-910.

74. Zhang X, Yan X, Gorman J, Hoffman SN, Zhang L, et al. (2014) Perioperative hyperglycemia is associated with postoperative neurocognitive disorders after cardiac surgery. Neuropsychiatric Disease and Treatment.

75. Slater JP, Guarino T, Stack J, Vinod K, Bustami RT, et al. (2009) Cerebral Oxygen Desaturation Predicts Cognitive Decline and Longer Hospital Stay After Cardiac Surgery. Ann Thorac Surg 87: 36-45.

76. Lin R, Zhang F, Xue Q, Yu B(2013) Accuracy of regional cerebral oxygen saturation in predicting postoperative cognitive dysfunction after total hip arthroplasy: regional cerebral oxygen saturation predicts POCD. The journal of Arthroplasty 28: 494-449.

77. Zheng F, Sheinberg R, Yee MS, Ono M, Zheng Y, et al. (2013) Cerebral near-infrared spectroscopy monitoring and neurologic outcomes in adult cardiac surgery patients: a systematic review. Anesthesia and Analgesia 116: 663-676.

78. Pugsley W, Klinger L, Paschalis C, Treasure T, Harrison M, et al. (1994) The impact of microemboli during cardiopulmonary bypass on neuropsychological functioning. Stroke 25: 1393-1399.

79. Knipp SC, Matatko N, Wilhelm H (2008) Cognitive outcomes three years after coronary artery bypass surgery: relation to diffusion-weighted magnetic resonance imaging. Annals of Thoracic Surgery 85: 872-879.

80. Liu YH, Wang DX, Li LH (2009) The effects of cardiopulmonary bypass on the number of cerebral microemboli and the incidence of cognitive dysfunction after coronary artery bypass graft surgery. Anesthesia and Analgesia 109: 1013-22.

81. Martin KK, Wigginton JB, Babikian VL (2009) Intraoperative cerebral high-intensity transient signals and postoperative cognitive function: a systematic review. American Journal of Surgery 197: 55-63.

82. Koch S, Forteza A, Lavernia C, Romano JG, Campo-Bustillo I, et al (2007) Cerebral fat microembolism and cognitive decline after hip and knee replacement. Stroke 38: 1079-1081.

83. Rodriguez RA, Tellier A, Grabowski J (2005) Cognitive dysfunction after total knee Arthroplasty: effects of Intraoperative cerebral Embolization and postoperative complications. The Journal of Arthroplasty 20: 763771.

84. Scott DA, Evered LA, Gerraty RP, MacIsaac A, Lai-Kwon J, et al. (2014) Cognitive dysfunction follows left heart catheterisation but is not related to microembolic count. Int J Cardiol 175: 67-71.

85. Marasco SF, Sharwood LN, Abramson MJ (2008) No improvement in neurocognitive outcomes after off-pump versus on-pump coronary revascularization: a meta-analysis. New England Journal of Medicine 33: 961-970.

86. Cormack F, Shipolini A, Awad WI, Richardson C, McCormack DJ, et al. (2012) A meta-analysis of cognitive outcome following coronary artery bypass graft surgery. Neurosci Biobehav Rev 36: 2118-2129.

87. Zhu J, Jiang X, Shi E, Ma H, Wang J (2009) “Sevoflurane preconditioning reverses impairment of hippocampal long-term potentiation induced by myocardial ischemia-reperfusion injury" European Journal of Anaesthesiology 26: 961-968. 
88. Vacas S, Degos V, Tracy K, Maze M (2014) “High-mobility Group Box Protein Initiates Postoperative Cognitive Decline by Engaging Bone Marrow-derived Macrophages.” Anesthesiology 120: 1160-1167.

89. Terrando N, Gómez-Galán M, Yang T, Carlström M, Gustavsson D, et al. (2013) Aspirin-triggered resolvin D1 prevents surgery-induced cognitive decline. FASEB J 27: 3564-3571.

90. Li SY, Xia LX, Zhao YL, Yang L, Chen YL, et al. (2013) Minocycline mitigates isoflurane-induced cognitive impairment in aged rats. Brain Res 1496: 84-93.

91. Vizcaychipi MP, Watts HR, O'Dea KP, Lloyd DG, Penn JW, et al. (2014) The therapeutic potential of atorvastatin in a mouse model of postoperative cognitive decline. Ann Surg 259: 1235-1244.

92. Zhang J, Tan H, Jiang W, Zuo Z (2014) "Amantadine Alleviates Postoperative Cognitive Dysfunction Possibly by Increasing Glial Cell Line-derived Neurotrophic Factor in Rats." Anesthesiology.

93. Sanders RD, Xu J, Shu Y, Januszewski A, Halder S, et al. (2009) Dexmedetomidine attenuates isoflurane-induced neurocognitive impairment in neonatal rats. Anesthesiology 110: 1077-1085.

94. Mathew J, White W, Shinderle D, Podgoreanu M, Berger M, et al. (2013) for the Neurologic Outcome Research Group (NORG) of The Duke Heart Center. "Intraoperative Magnesium Administration Does Not Improve Neurocognitive Function After Cardiac Surgery.” Stroke.

95. Hudetz J, Iqbal Z, Gandhi SD, Patterson KM, Byrne AJ, et al. (2009) "Ketamine attenuates post-operative cognitive dysfunction after cardiac surgery." Acta Anesthesiol Sanda 53: 864-872.

96. Schricker T, Sato H2, Beaudry T3, Codere T1, Hatzakorzian R1, et al (2014) Intraoperative maintenance of normoglycemia with insulin and glucose preserves verbal learning after cardiac surgery. PLoS One 9: e99661.

97. Chen Y, Run X, Liang Z, Zhao Y, Dai C, et al. (2014) "Intranasal insulin prevents anesthesia-induced hyperphosphorylation of tau in $3 \times \mathrm{Tg}$ ADmice ." Frontiers in Aging Neuroscience.

98. Hansen M, Madsen M, Anderson L, Hageman I, Rasmussen LS, et al. (2014) "Effect of Melatonin on Cognitive Function and Sleep in relation to Breast Cancer Surgery: A Randomized, Double-Blind, PlaceboControlled Trial." International Journal of Breast Cancer.

99. Fang Q, Qian X, An J, Wen H, Cope DK, et al. (2014) Higher dose dexamethasone increases early postoperative cognitive dysfunction. J Neurosurg Anesthesiol 26: 220-225.

100. Ottens TH, Dieleman JM, Sauër AM, Peelen LM, Nierich AP, et al. (2014) Effects of dexamethasone on cognitive decline after cardiac surgery: a randomized clinical trial. Anesthesiology 121: 492-500.

101. Valentin L, Monk T, Pietrobon R, Nielsen K, Vissoci J, et al. (2014) "Effects of Dexamethasone and Depth of Anesthesia on the incidence of Postoperative Cognitive Dysfunction in Elderly Patients Undergoing Non-Cardiac Surgery- A Double Blinded RCT.” Abstract submitted to ASA.

102. Papadopoulos G, Pouangare M, Papathanakos G, Arnaoutoglou E, Petrou A, et al. (2014) The effect of ondansetron on postoperative delirium and cognitive function in aged orthopedic patients. Minerva Anestesiol 80: 444-451.

103. Kawano T, Takahashi T, Iwata H, Morikawa A, Imori S, et al. (2014) Effects of ketoprofen for prevention of postoperative cognitive dysfunction in aged rats. J Anesth 28: 932-936.

104. Li Z, Cao Y, Li L, Liang Y, Tian X, et al. (2014) "Prophylactic angiotensin type 1 receptor antagonism confers neuroprotection in an aged rat model of postoperative cognitive dysfunction.” Biochemical and Biophysical Research Communications 449: 74-80.

105. Li X, Zhou M, Wang X, Ji M, Zhou Z, et al. (2014) "Resveratrol Pretreatment Attenuates the Isoflurane-Induced Cognitive Impairment Through its Anti-Inflammation and -Apoptosis Actions in Aged Mice." Journal of Molecular Neuroscience. 52: 286-293.

106. Bilotta F, Gelb AW, Stazi E, Titi L, Paoloni FP, et al. (2013) Pharmacological perioperative brain neuroprotection: a qualitative review of randomized clinical trials. Br J Anaesth 110 Suppl 1: i113-120.

107. Rortgen D, Kloos J, Fries M, Grottke O, Rex S, et al. (2010) "Comparison of early cognitive function and recovery after desflurane or sevoflurane anaesthesia in the elderly: a double-blinded randomized controlled trial." British Journal of Anaesthesia $104: 167-74$.

108. Zhang Y, Xu Z, Wang H, Dong Y, Shi HN, et al. (2012) Anesthetics isoflurane and desflurane differently affect mitochondrial function, learning, and memory. Ann Neurol 71: 687-698.

109. Zhang B, Tian M, Zhen Y, Yue Y, Sherman J, et al. (2012) The effects of isoflurane and desflurane on cognitive function in humans. Anesth Analg 114: 410-415.

110. Shen X, Dong Y, Xu Z, Wang H, Miao C, et al. (2013) "Selective Anesthesia-induced Neuroinflammation in Developing Mouse Brain and Cognitive Impairment." Anesthesiology 118: 3.

111. Arora SS, Gooch JL, García PS (2014) Postoperative cognitive dysfunction, Alzheimer's disease, and anesthesia. Int J Neurosci 124 236-242.

112. Rasmussen LS, Steentoft A, Rasmussen H, Kristensen PA, Moller JT (1999) Benzodiazepines and postoperative cognitive dysfunction in the elderly. ISPOCD Group. International Study of Postoperative Cognitive Dysfunction. Br J Anaesth 83: 585-589.

113. Morandi A, Brummel NE, Ely EW (2011) Sedation, delirium and mechanical ventilation: the 'ABCDE' approach. Curr Opin Crit Care 17: 43-49.

114. Deiner S, Silverstein JH (2009) Postoperative delirium and cognitive dysfunction. Br J Anaesth 103 Suppl 1: i41-46.

115. Girard TD, Jackson JC, Pandharipande PP, Pun BT, Thompson JL, et al. (2010) Delirium as a predictor of long-term cognitive impairment in survivors of critical illness. Crit Care Med 38: 1513-1520.

116. Koster S, Hensens AG, van der Palen J (2009) The long-term cognitive and functional outcomes of postoperative delirium after cardiac surgery. Ann Thorac Surg 87: 1469-1474.

117. Liang C, Chu C, Chou M, Lin Y, Lu T, et al. (2014) "Interrelationship of postoperative delirium and cognitive impairment and their impact on the functional status in older patients undergoing orthopaedic surgery: A prospective cohort study." PLOS ONE 9: 1-8.

118. Morandi A, Rogers B, Gunther M, Merkle K, Pandharipande P, et al. (2012) "The relationship between delirium duration, white matter integrity, and cognitive impairment in intensive care unit survivors as determined by diffusion tensor imaging: The VISIONS prospective cohort magnetic resonance imaging study." Critical Care Medicine 40: 2182-2189.

119. Vacas S, Degos V, Feng X, Maze M (2013) The neuroinflammatory response of postoperative cognitive decline. Br Med Bull 106: 161-178.

120. Krenk L, Kehlet H, Bæk Hansen T, Solgaard S, Soballe K, et al. (2014) Cognitive dysfunction after fast-track hip and knee replacement. Anesth Analg 118: 1034-1040. 